\title{
DISCUSSION: Rotation in Relation with Abundances and Magnetic Fields
}

\author{
Sylvie Vauclair \\ Observatoire Midi-Pyrénées, Toulouse, France
}

\begin{abstract}
This general discussion was open on the papers presented in oral session II and related posters.
\end{abstract}

The following subjects were proposed for the discussion :

\section{Seismic Tests of Rotation and Abundances in the Sun and Stars}

Sylvie Vauclair: The importance of future seismic tests of stellar internal parameters has to be stressed. Several groups are now working to prepare the space missions which have been described in the previous session (COROT, MOST, EDDINGTON). For example we develop a group working on the possible tests of diffusion processes in stars. We compute several models of a star with the same external parameters ( $L, T_{\text {eff }}$, outer chemical composition) but with a different history: one without any diffusion, one with pure element settling, others with settling and some mixing. The models are iterated to obtain the final stage with same external parameters. We then compare the oscillation frequencies of the various models. It seems that diffusion may have a specific and possibly detectable signature in the so-called "normal stars" (Théado, Charpinet \& Vauclair, in preparation).

Juri Toomre : Could you expand on the properties of roAp stars that now yield diagnostic possibilities?

Jaymie Matthews : the fundamental frequency spacing $\Delta \nu$ leads to $L$ if $T_{\text {eff }}$ is known (assuming small mass uncertainty); the spacing of the frequency multiplets gives the rotation frequency $\Omega$; the relative amplitude of the sidelobes is related to $\Omega^{2} / B$; the small frequency spacing $\delta \nu_{02}$ is sensitive to the composition of the convective core and to the internal $\mathrm{B}$ field; the oscillation amplitudes give access to atmospheric dynamics; the maximum frequency below acoustic cut-off is related to $T(\tau)$ in the atmosphere (all this represents work in progress).

\section{Uncertainties on Abundance Measurements and Rotation}

Sylvie Vauclair : The abundances of light elements still represent important constraints on the stellar internal structure. In the Sun, for example, it is difficult to account for the sound velocity determined from helioseismology with helium settling and mixing and keep normal beryllium abundance. Could you comment on the present uncertainties on the lithium and beryllium determinations in stars. 
Suchitra Balachandran : I confirm that beryllium is not depleted in the Sun when one takes into account correctly the UV opacity.

Dainis Dravins : While lithium abundances indeed are important for probing the history of stellar interiors, their accurate determination involves several uncertainties due to mainly the temperature dependence of the main Li I line. A striking example is that its equivalent width in sunspots is two orders of magnitude greater than in the quiet-sun photosphere. Thus, stellar line strengths may depend on the character of stellar surface inhomogeneities. Since these are expected to be different in low metallicity stars, there is a risk that such changes in stellar granulation may mimic a changed lithium abundance. A related question is that of the isotopic ratio ${ }^{6} \mathrm{Li} /{ }^{7} \mathrm{Li}$, in principle observable as a slight asymmetry of the $\mathrm{Li}$ I line. However, that must be segregated from an analogous asymmetry caused by the asymmetric velocity patterns in stellar surface convection. These topics are being discussed in the literature (including also the possible effects of three-dimensional radiative transfer, and others), and it seems likely that a better understanding will be gained in the near future.

Jean-Paul Zahn : What is the rotational history of pop II stars?

Marc Pinsonneault : We have limited data on the rotation of Pop II stars. We know that there are a significant number of rapidly rotating horizontal branch stars, with a range of $v \sin i$ up to $40 \mathrm{~km} . \mathrm{s}^{-1}$ and interesting trends with $T_{\text {eff. }}$ On the main sequence, single stars are sharp line objects with $v \sin i<8$ $\mathrm{km} \mathrm{s}^{-1} \cdot{ }^{6} \mathrm{Li} /{ }^{7} \mathrm{Li}$ isotope studies at high resolution and $\mathrm{S} / \mathrm{N}$ ratios show evidence for extra line broadening at the $4-5 \mathrm{~km} \mathrm{~s}^{-1}$ level.

Sylvie Vauclair : I agree : Population II stars rotate slowly but we do not know their rotational history. For their modelisation, some people assume that their rotation history was the same as for the Sun, but it is also possible to assume that they have always rotated slowly.

Douglas Gies : Is it possible to have synchronisation of a solar type star with a Jupiter-like planet?

Gibor Basri : The synchronization of a star by a planet will only happen if the orbital period of the planet is about 3 days or less. This is seen in the $\tau$ Boo system (with a lower limit on the mass of the planet of about $3 \mathrm{M}_{\text {Jupiter }}$.

Jean Zorec : I would like to give a comment about Be stars. We have studied five pole-on Be stars with local one dimensional non-LTE models in rotationally distorted stars having gravitationally darkened atmospheres; in a plot of $\mathrm{C} / \mathrm{N}$ ratios against oxygen abundance, the $\mathrm{C} / \mathrm{N}$ ratios of $\mathrm{Be}$ stars were compared with those of slowly rotating $\mathrm{B}$ stars without emission studied by Gies and Lambert (1992, ApJ 387, 673) and with the limiting ratios predicted by Meynet and Maeder's (2000, A\&A 361, 101) models of rotating stars for their main sequence end. The $\mathrm{C} / \mathrm{N}$ abundance ratios of $\mathrm{Be}$ stars found are systematically lower than observed for slowly rotating B stars without emission. They imply that carbon is somewhat depleted, while nitrogen is enhanced, as expected from mixing induced by fast rotation.

\section{Magnetic Fields and Doppler Imaging}

Sylvie Vauclair : Now let us open a discusison about doppler imaging and magnetic fields as presented in this session by J.-F. Donati and P. Petit. 
Ilkka Tuominen : Doppler imaging, as the name already tells, is based on the Doppler effect: line profile variations over the rotation period, like bumps moving from the blue side to the red side of the line profile when the star rotates. A polar cap does not produce any variations to the line profile. The profile variations are inverted with respect to a temperature map comparing to some reference line profile, e.g. theoretical local line profiles over the disk. If the average observed profile has some systematic difference compared to the reference profile, the inversion produces artifacts to the image, like polar caps. That is why the local line profiles have to be derived carefully, taking into account effects like limb darkening in the line and continuum, blends, microand macroturbulence.

Concerning the inversion of the magnetic field vector, the circular polarization (Stokes V) gives only the longitudinal component of the field. Donati claims that their inversions give the azimutal field because it is seen longitudinal at the limb. This would need however, I guess, zero noise observations (infinite $\mathrm{S} / \mathrm{N}$ ) and continuous phase coverage over the rotational period. In addition, certainly the Stokes V and I depend on each other (e.g. according to Unno's equations), in other words magnetic field has influence on temperature and temperature on magnetic field. They cannot be solved independently, and hence the polarized line profiles should be calculated in detail.

Jean-Francois Donati : Brightness and magnetic imaging of rapidly rotating cool active stars has been the subject of quite a large number of studies published in the refereed literature in the last decade. These techniques are now known to work pretty well, if we keep in mind their inherent limitations. For instance, the spatial resolution we can obtain close to the stellar equator is usually much worse in latitude than in longitude, which makes low latitude features on reconstructed stellar images look very elongated in latitude, considerably more than what they probably are at the surface of the star itself.

However, a number of older issues, on which concerns were raised in the past, were addressed in a very detailed way by several authors and do no longer need to be considered as a potential source of problem.

While the reality of polar spots was discussed extensively at various workshops and in several literature papers in the last decade, all other attempts at explaining the constantly flat bottomed profiles exhibited by most active stars have essentially failed up to now. The most convincing evidence remains the fact that stars with polar spots are sometimes found (even if only rarely) with no such polar spots, demonstrating clearly that this specific spectral signature attributed to polar spots is indeed due to a time variable phenomenon at the surface of the star, and not to a wrong modelling of the stellar lines.

As far as imaging magnetic fields is concerned, it has been now established in several independant simulations papers, carried out with independant imaging codes, that certain distributions of magnetic fields, and in particular those found in cool active stars, can be well recovered, even from Stokes V data sets only. In particular, these simulations show that azimuthal magnetic fields at the surface of the star can be very clearly distinguished from radial or meridional magnetic fields, from the very different signatures and rotational modulation they can produce in stellar spectra. All this is published in the refereed literature, and available for whoever is interested on the subject. 